\title{
DIVERSIFICACIÓN DE QUESOS DE CABRA: EL USO DE ACEITES DE OLIVA VIRGEN EXTRA MONOVARIETALES (PÓSTER)
}

\author{
$\underline{\text { Luis Pablo Ureña }}^{a^{*}}$, Sergio Colombo ${ }^{a}$, María José Sánchez ${ }^{b}$, Thais Rucabado ${ }^{b}$ Enrique Cazorla $^{b}$, \\ Angel Luis López ${ }^{c}$, Francisco de Asís Ruiz ${ }^{a}$. \\ a IFAPA Centro Camino de Purchil-Junta de Andalucía (Granada, luisp.urena@ juntadeandalucia.es). \\ ${ }^{b}$ Agrupación de Ganaderos de los Montes de Málaga- AGAMMA SCA. ${ }^{c}$ IFAPA Centro Hinojosa del \\ Duque-Junta de Andalucía
}

Palabras clave: lácteos, comercialización, quesería, conservación.

\section{Introducción}

En el contexto actual, debido tanto a los condicionantes económicos como las vicisitudes sociosanitarias, las industrias lácteas más que nunca están buscando diferenciar sus productos, para dar respuesta así a un consumidor más concienciado y preocupado por lo que come y en un mercado cada vez más competitivo. De ahí que el sector quesero esté trabajando para dar respuesta a estas demandas mediante el incremento de especialidades elaboradas (enzimáticas, lácticas...), el uso de aditivos en corteza (pimentón, tomillo, romero, orégano...), la certificación en ecológico, el uso del logotipo de raza autóctona o, más recientemente, el uso de leche certificado como de pastoreo, entre otros.

Teniendo esto en cuenta, partimos de la base de que en Andalucía ya existe una larga tradición de uso del aceite de oliva en la elaboración de quesos. Este uso tradicional daba respuesta tanto a la necesidad de conservar este producto lácteo, otorgándole así una mayor vida útil sin necesidad de temperaturas de refrigeración, como también a la búsqueda de potenciar algunos de sus atributos sensoriales (cremosidad y untuosidad) y aportar complejidad aromática (notas frutales, picantes...).

\section{Objetivo}

Aprovechando este corpus de conocimiento previo del sector quesero andaluz, y dando un paso más en cuanto a la diferenciación del producto, el objetivo de este estudio ha sido evaluar tres aceites de oliva virgen extra monovarietales: Verdial de Málaga, Hojiblanca y Picual, en la elaboración de queso de cabra en aceite. Con estos resultados se trabajará posteriormente con expertos y consumidores para su valoración sensorial.

\section{Metodología}

La experimentación se ha llevado a cabo en la planta de lácteos de Agrupación de Ganaderos de los Montes de Málaga (AGAMMA), situadas en Colmenar (Málaga), partiendo de sus procedimientos habituales en la fabricación de queso de cabra madurados en aceite de oliva. Así, se seleccionaron 3 aceites monovarietales de la zona, de categoría virgen extra: Verdial, Hojiblanca y Picual. El queso usado fue un queso curado de cabra de leche pasteurizada, con entre 2- 3 meses de maduración. Durante 9 meses, se sumergieron en cada aceite 8 quesos $\left(1 \mathrm{~kg}\right.$.) en contenedores alimentarios opacos con 20 litros de aceite cada uno (12-14 ${ }^{\circ} \mathrm{C}$; HR $85-90 \%$ ) durante 3 meses, repitiendo el proceso hasta un total de tres veces. Se tomaron muestras mensuales de los aceites (50 ml.), para un total de 33 muestras, que se conservaron a temperaturas de congelación ($\left.40^{\circ} \mathrm{C}\right)$.

Se realizó un análisis físico-químico de los aceites, de los siguientes parámetros: acidez, índice de peróxidos, humedad e impurezas, en laboratorio certificado (Reglamento (CEE) $n^{\circ}$ 2568/91) con reconocimiento del Consejo Oleícola Internacional (COI). Además, se ha analizado la evolución del color en la corteza del queso a la salida del aceite en cada uno de los tres lotes de quesos usados. El procedimiento usado fue la toma de 10 mediciones en corteza, mediante el uso de un colorímetro triestímulo Konica Minolta CR-400, previa calibración con patrón blanco. La toma de datos se realizó mediante el espacio de color $\mathrm{L}^{*} \mathrm{a} * \mathrm{~b} *$ (CIELAB), haciendo las representaciones gracias al programa freeware "Colour Conversion Centre". A los datos obtenidos se les ha realizado un análisis estadístico, mediante técnicas descriptivas e inferenciales, empleando el paquete de software IBM SPSS Statistics 25. 


\section{Resultados}

Atendiendo al resumen de la analítica física-química de los aceites (Cuadro 1); podemos ver a priori, como la variedad Hojiblanca ha sido la que ha evolucionado en una mayor acidez (media más alta), siendo lo contrario no obstante para el caso de los peróxidos (menor cantidad de media) y encontrando diferencias muy leves para el caso de la humedad e impurezas. Al profundizar en el análisis estadístico de los datos, primero comprobamos la normalidad de la distribución de los datos mediante la prueba de Shapiro Wilk, y la homocedasticidad por la prueba de Levene. Una vez confirmados ambos supuestos, procedimos a realizar test ANOVA de una vía. Así, y para un nivel de significación de 0,05 ( $\mathrm{F}=109,90 ; \mathrm{p}=0,00)$ encontramos diferencias significativas entre las variedades únicamente para la medición de la cantidad de peróxidos. Analizando estas diferencias con un análisis post hoc (test de Bonferroni), encontramos que con una probabilidad de 0,00 y 0,42 respectivamente $(\alpha=0,05)$, el aceite usado de la variedad Hojiblanca tiene de media menor cantidad de peróxidos que la Picual (muy significativamente) y que la Verdial.

Cuadro 1. Media aritmética y error estándar de los parámetros laboratoriales, para las tres variedades de aceite.

\begin{tabular}{|c|c|c|c|c|c|c|c|c|}
\hline & \multicolumn{2}{|c|}{ Acidez } & \multicolumn{2}{c|}{ Peróxidos } & \multicolumn{2}{c|}{ Humedad } & \multicolumn{2}{c|}{ Impurezas } \\
\cline { 2 - 9 } & Media & Error std. & Media & Error std. & Media & Error std. & Media & Error std. \\
\hline Hojiblanca & 9,99 & 1,70 & 4,56 & 0,18 & 0,12 & 0,01 & 0,08 & 0,01 \\
\hline Picual & 9,59 & 1,36 & 11,33 & 0,48 & 0,12 & 0,01 & 0,10 & 0,01 \\
\hline Verdial & 9,32 & 1,06 & 5,83 & 0,30 & 0,14 & 0,02 & 0,09 & 0,02 \\
\hline TOTAL & 9,63 & 0,78 & 7,24 & 0,55 & 0,13 & 0,01 & 0,09 & 0,01 \\
\hline
\end{tabular}

En cuanto al color del queso (Cuadro 2), Si tenemos en cuenta que, en el espacio de color CIELAB, la variable $* \mathrm{~L}$ representa la luminosidad, y las variables *a y $* \mathrm{~b}$ son las coordenadas cromáticas, podemos simplificar: *a desde rojo (+a) a verde $(-a)$; *b desde amarillo $(+b)$ a azul $(+b)$, se observa progresivamente del lote 1 al 3 una pérdida de la luminosidad del color y una tendencia hacia tonos menos rojos y más amarillos.

Cuadro 2. Media aritmética y error estándar de los parámetros del espacio de color $L^{*} a * b(C I E L A B)$ para la corteza de los quesos.

\begin{tabular}{|c|c|c|c|c|c|c|}
\hline \multirow{2}{*}{} & \multicolumn{5}{|c|}{ LOTE 1: FEBRERO } \\
\cline { 2 - 7 } & \multicolumn{2}{|c|}{ L* $^{*}$} & \multicolumn{2}{|c|}{ a $^{*}$} & \multicolumn{2}{c|}{ b $^{*}$} \\
\cline { 2 - 7 } & Media & Error std. & Media & Error std. & Media & Error std. \\
\hline Hojiblanca & 56,71 & 0,74 & 30,40 & 0,51 & 69,06 & 1,23 \\
\hline Picual & 61,67 & 0,69 & 31,78 & 0,44 & 78,54 & 0,69 \\
\hline Verdial & 52,55 & 0,37 & 30,79 & 0,27 & 56,90 & 0,76 \\
\hline
\end{tabular}




\begin{tabular}{|c|c|c|c|c|c|c|}
\hline & \multicolumn{6}{|c|}{ LOTE 2: MAYO } \\
\hline & \multicolumn{2}{|c|}{$\mathbf{L}^{*}$} & \multicolumn{2}{|r|}{ a* } & \multicolumn{2}{|c|}{$\mathbf{b}^{*}$} \\
\hline & Media & Error std. & Media & Error std. & Media & Error std. \\
\hline Hojiblanca & 72,30 & 0,68 & 27,66 & 0,55 & 89,07 & 0,84 \\
\hline Picual & 73,25 & 0,80 & 28,34 & 0,76 & 90,86 & 0,71 \\
\hline \multirow[t]{4}{*}{ Verdial } & 69,13 & 1,95 & 23,14 & 1,05 & 86,74 & 0,68 \\
\hline & \multicolumn{6}{|c|}{ LOTE 3: SEPTIEMBRE } \\
\hline & \multicolumn{2}{|c|}{$\mathbf{L}^{*}$} & \multicolumn{2}{|r|}{$\mathbf{a}^{*}$} & \multicolumn{2}{|c|}{$\mathbf{b}^{*}$} \\
\hline & Media & Error std. & Media & Error std. & Media & Error std. \\
\hline Hojiblanca & 75,95 & 0,34 & 24,04 & 0,26 & 89,88 & 0,64 \\
\hline Picual & 75,21 & 0,92 & 27,34 & 0,56 & 92,05 & 1,16 \\
\hline Verdial & 73,15 & 0,70 & 23,36 & 0,47 & 90,00 & 2,10 \\
\hline
\end{tabular}

Profundizando en los datos en la búsqueda de posibles diferencias entre los valores medidos, se ha realizado un análisis ANOVA junto con test post hoc de Tukey (Cuadro 3). Así, y para un nivel de significación de 0,05 , para la variable de color $\mathrm{L}^{*}(\mathrm{~F}=1,625 ; \mathrm{p}=0,203)$ no encontramos diferencias significativas, pero sí para el caso de las variables $a^{*}(\mathrm{~F}=3,612 ; \mathrm{p}=0,031)$ y $\mathrm{b}^{*}(\mathrm{~F}=18,6938 ; \mathrm{p}=0,000)$. Analizando estas diferencias con un análisis post hoc (test de Tukey), encontramos que $\mathrm{a}^{*}$ es menor en el aceite Picual que en el Hojiblanca $(\mathrm{p}=0,024)$, y que $\mathrm{b}^{*}$ es mayor en Picual que para el aceite Verdial $(\mathrm{p}=0,000)$ y que para el Hojiblanca $(0,000)$.

Cuadro 3. Media y desviación estándar de las variables de color: Letras diferentes en la misma columna indican diferencias significativas $(p<0.05)$ según test de Tukey.

\begin{tabular}{|c|c|c|c|c|c|c|}
\hline \multirow{2}{*}{} & \multicolumn{2}{|c|}{ L* $^{*}$} & \multicolumn{2}{c|}{ a* $^{*}$} & \multicolumn{2}{c|}{ b $^{*}$} \\
\cline { 2 - 7 } & Media & Desv. std. & Media & Desv. std. & Media & Desv. std. \\
\hline Hoijblanca & $66,9330 a$ & 10,56128 & $5,7603 b$ & 8,00576 & $25,8063 b$ & 1,93649 \\
\hline Picual & $70,0450 a$ & 6,55891 & $1,6633 a$ & 3,64921 & $28,9023 a$ & 2,43909 \\
\hline Verdial & $66,3300 a$ & 8,09958 & $3,9880 a b$ & 5,27090 & $24,9193 b$ & 3,32886 \\
\hline TOTAL & 67,7693 & 8,62660 & 3,8039 & 6,09278 & 26,5427 & 3,11754 \\
\hline
\end{tabular}

Se ha realizado además un análisis de Componentes Principales, mediante test KMO y Bartlett $(0,515$; $\mathrm{p}=0,000$ ), en el que se extrajeron dos componentes explicando el $97,44 \%$ de la varianza total. Según está representado en el Gráfico 1, el primer componente (eje X) explica el 61,92\% de la varianza, y muestra una correlación positiva con la variable de color a*, y el segundo componente (eje Y) explica el 31,52 \% restante y muestra una correlación positiva con la variable b*. 
Gráfico 1. Representación en dos factores del análisis de componentes principales (Test KMO y Barlett).

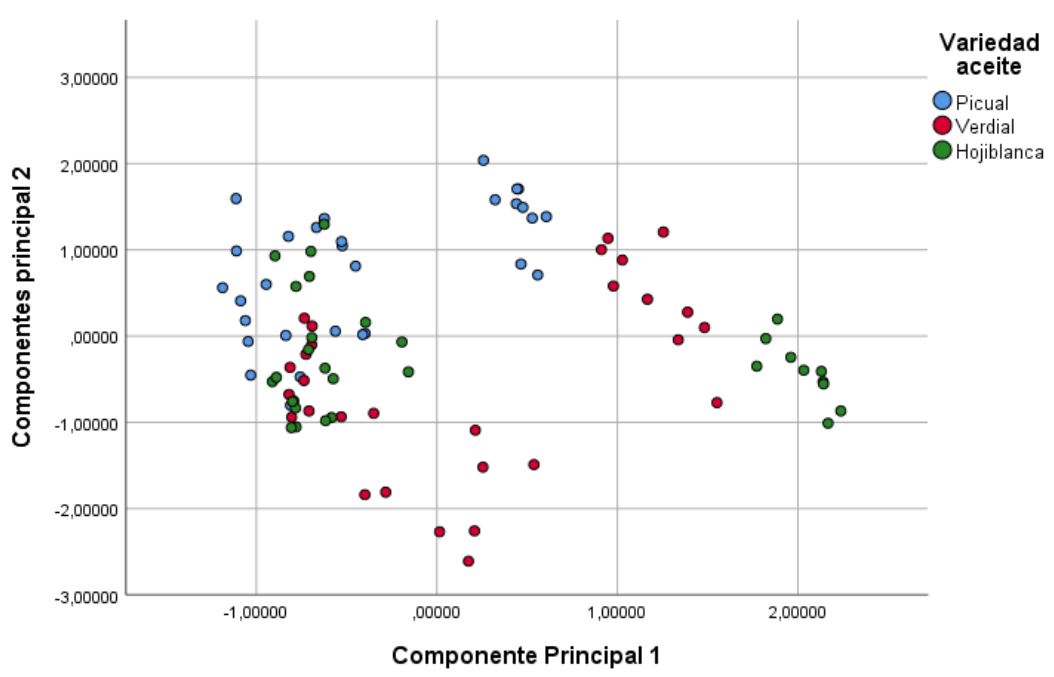

\section{Conclusiones}

De los resultados obtenidos, se concluye que existe una evolución creciente de los parámetros: i) acidez y ii) humedad de los aceites con el tiempo de uso en la maceración del queso. Así mismo, se encuentran diferencias con respecto al contenido de peróxidos, que al mantenerse durante todo el estudio pueden deberse al estado inicial de conservación y calidad de los aceites usados. Las condiciones de almacenamiento específicas de la maceración de queso en aceite (baja temperatura y humedad alta), siguiendo a autores como Gutiérrez et. al (2002), nos pueden indicar que los aceites aún están en periodo de inducción, y que para encontrar mayores diferencias en los parámetros fisicoquímicos de los aceites sería necesario ampliar el tiempo de experimentación en futuros estudios.

Además, se han obtenido coloraciones más rojas y oscuras en la corteza de los quesos sumergidos, con diferencias según la variedad de aceite virgen extra usada y más acusadas en el caso del aceite de la variedad Verdial. Esto se diluye a partir del segundo lote de queso, y por tanto indicaría que existe una cierta retirada de pigmentos provenientes del aceite o una modificación de su biodisponibilidad, como sería el caso de los carotenoides según Meléndez-Martínez et al., 2020. Estas diferencias en la coloración son de gran interés para la industria quesera, y por tanto actualmente se está desarrollando otro estudio en el que se están explorando y ampliando los resultados obtenidos en el presente trabajo.

\section{Bibliografía:}

Reglamento (CEE) n ${ }^{\circ}$ 2568/91, de 11 de julio. (1991). "On the characteristics of Olive Oil and Olive residue Oil and the relevant Methods of analysis". Official Journal of the European Communities, $\mathrm{n}^{\circ} \mathrm{L} 248$.

Gutiérrez, F., Villafranca, M.J., y Castellano, J.M. (2002). "Changes in the main components and quality indices of virgin olive oil during oxidation". Journal of the American Oil Chemists' Society, 79(7):669-676.

Meléndez-Martínez, A.J., Mandić, A.I., Bantis, F., Böhm, V., Borge, G.I.A., Brnčić, M., Bysted, A., Cano, M.P., Días, M.G., Elgersma, A., Fikselová, M., García-Alonso, J., Kljak, K., Lavelli, V., Manganaris, G.A., Mapelli-Brahm, P., Marounek, M., Olmedilla-Alonso, B., Periago-Castón, M.J., Pintea, A., Sheehan, J.J., Saponjac, V.T., Valkisova-Frey, M., Van Meulebroek, L. y O’Brien, N. (2020). "A comprehensive review on carotenoids in foods and feeds: status quo, applications, patents, and research needs". Critical Reviews in Food Science and Nutrition, 1-51.

Agradecimientos: Esta investigación forma parte del proyecto Transforma "Retos de los sistemas ganaderos andaluces y sus productos (RESGAP)" (2019-2021), financiado por el Fondo Europeo de Desarrollo Regional, dentro del Programa Operativo FEDER de Andalucía 2014-2020. 